\title{
Hot atom chemistry: oxygen at stepped platinum surfaces
}

\author{
Axel Groß \\ Institute of Theoretical Chemistry, Ulm University, 89069 Ulm/Germany and \\ Helmholtz Institute Ulm (HIU) Electrochemical Energy Storage, 89081 Ulm/Germany
}

\begin{abstract}
It is a paradigm in chemistry that chemical reaction are mainly governed by thermodynamics. Within this assumption, reaction rates can be derived from transition state theory which requires a quasi-equilibrium between reactants and activated transition state complexes that is achieved through friction. However, to reach thermal equilibrium through friction takes some time. Here we show, based on ab initio molecular dynamics simulations of the interaction of molecular oxygen with stepped Pt surfaces, that chemical reactions in heterogeneous catalysis can occur in a nonequilibrium fashion when the excess kinetic energy upon entering the potential well of a reaction intermediate is large enough.
\end{abstract}

\section{INTRODUCTION}

Our basic understanding of chemical reactions in the presence of a catalyst is based on thermodynamic concepts. Complex reactions can consist of several elementary reaction steps which are each characterized by a reactant and a product state that are typically separated by an activation barrier. Usually it is assumed that the redistribution of excess kinetic energy is much faster than the propagation from one reaction intermediate to the next [1]. Then chemical reactions occur at a characteristic reaction rate at a given temperature and chemical concentration that can be determined using transition state theory (TST) [2-5]. Its application demands a quasiequilibrium between reactants and activated transition state complexes.

Reaching equilibrium has to be achieved through friction [4], i.e., through a strong coupling with the corresponding heat bath. This represents a crucial assumption underlying transition state theory. If their is only low-to-moderate coupling, transition state theory cannot be applied without refinement, as has already been discussed in the seminal work of Kramers [6]. Deviation from TST behavior have already been discussed for organic reaction intermediates [1]. Furthermore, so-called "hot atoms" dynamics have been invoked to explain experimental observations in the spatial distribution of dissociation products on surfaces $[7,8]$. On $\mathrm{Al}(111)$, according to scannning tunneling microscopy (STM) experiments, a small dosage of molecular oxygen exclusively leads to single oxygen atoms separated by an average distance of more than $80 \AA$ [7]. Various mechanisms have been proposed to explain this surprising results. For example, subsurface migration of single oxygen atoms has been invoked [9]. However, also a hot atom-like mechanism based on a cannonball abstraction process $[10,11]$ has been proposed. First-principles based molecular dynamics simulations including spin selection rules [12, 13] have been able to reproduce and explain the experimentally observed samm sticking probability at low kinetic energies [14, 15], but have not found any indications for the cannonball mechanism. On Pt(111), molecular chemisorbed $\mathrm{O}_{2}$ molecules dissociate at higher temperatures with the oxygen atoms being preferentially to lattice sites apart from each other [8] which has also been explained by a hot atom mechanism and confirmed in kinetic Monte Carlo simulations [16].

However, the direct identification of hot atom dynamics in reactions on surfaces by a combination of experiment and theory is hard to achieve. From an experimental point of view this is due to the fact that the lifetime of dynamical hot atom events is typically so short that these events can not be directly detected. As far as simulations are concerned, in recent years significant progress has been made by performing dynamical simulations that include the coupling either to a phonon bath [15, 17-21] or to electron-hole pairs [22-24]. Such simulations have provided very valuable insights into hot atom dynamics in reactions Gambardella2001 at surfaces. Still it is fair to say that there is not yet a general understanding about the factors promoting hot atom reactions at surfaces.

In this study, I will demonstrate that hot atom dynamics plays a critical role in the interaction of $\mathrm{O}_{2}$ molecules with stepped Pt surfaces. The adsorption and dissociation of oxygen on platinum surfaces are of significant technological importance, as they not only constitute crucial reaction steps in the car exhaust catalyst [25], but they are also relevant for the oxygen reduction reaction in fuel cells in aqueous environments [26-30]. Molecular oxygen can adsorb both molecularly and dissociatively on $\operatorname{Pt}(111)$ [8, 31]. At low surface temperatures, however, oxygen does not directly dissociate on $\operatorname{Pt}(111)$, even if impinging with high kinetic energies $[8,32,33]$. Rather, it first adsorbs molecularly due to steric hindrance caused by the shape of the underlying potential energy surface, and only in a second step dissociates caused by thermal fluctuations, as demonstrated in tight-binding molecular dynamics studies [34, 35].

Low coordinated sites on catalytic surfaces are known to enhance the catalytic activity [36-39] due to the presence of low-coordinated sites which typically interact strongly with adsorbates. In this context, also the interaction of oxygen with stepped surface has raised a lot 


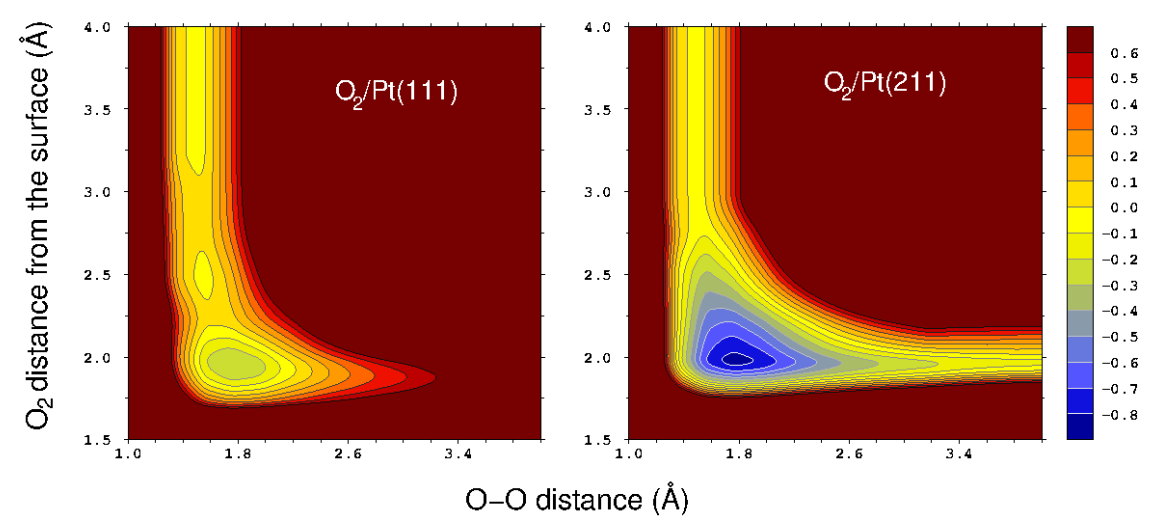

FIG. 1. Two-dimensional cuts through the six-dimensional potential energy surfaces of $\mathrm{O}_{2} / \mathrm{Pt}(111)$ and $\mathrm{O}_{2} / \mathrm{Pt}(211)$ as a function of the $\mathrm{O}-\mathrm{O}$ distance and the $\mathrm{O}_{2}$ center of mass distance from the surface in a t-b-t geometry (see text). On Pt(211), the $\mathrm{O}_{2}$ center of mass is directly above the atoms at the upper side of the steps with the molecular axis being parallel to the steps. The contour spacing in $0.1 \mathrm{eV}$.

of interest [40-46]. A joint experimental and theoretical study revealed that $\mathrm{O}_{2}$ dissociation is strongly favored at the step sites [42]. DFT calculations found that the adsorption energies of oxgen atoms and molecules at $\mathrm{Pt}$ step sites is signficantly enhanced compared to terrace sites $[42,47,48]$. However, interestingly enough the DFT calculations also showed that the enhanced $\mathrm{O}_{2}$ dissociation is not caused by a lowering of the local dissociation barrier $[42,47]$. The exact dissociation $\mathrm{O}_{2}$ mechanism at the step sizes could not be revealed in these studies, but it was speculated that it still proceeds as a thermal equilibrium process rather than through a hot precursor [42]. Here I will provide evidence based on $a b$ initio molecular dynamics simulations (AIMD) of $\mathrm{O}_{2}$ adsorption on stepped $\mathrm{Pt}$ surfaces that it is indeed a hot atom or rather a hot precursor mechanism that contributes to the preferential $\mathrm{O}_{2}$ dissociation on stepped Pt surfaces.

\section{COMPUTATIONAL DETAILS}

In this work, the dynamics of oxygen adsorption on flat and stepped Pt surfaces has been addressed by performing ab initio molecular dynamics simulations on the basis of density functional theory (DFT). The whole adsorption process including dissociation events and the accommodation of the hot atoms has been treated in a fully consistent manner. The periodic DFT calculations have been performed using the Vienna $a b$ initio simulation package (VASP) [49] in a spin-polarized fashion. The exchange-correlation effects have been described within the generalized gradient approximation (GGA) using the RPBE functional [50], as this functional yielded sticking probabilities of $\mathrm{O}_{2} / \mathrm{Pt}(111)$ [51] in better agreement with the experiment than the PBE functional [52]. The one-electron valence states were expanded in plane waves with kinetic energies up to the cutoff energy of $400 \mathrm{eV}$, and the ionic cores were represented by projector augmented wave (PAW) potentials [53] as constructed by Kresse and Joubert [54]. The Pt(111) surface was modeled by a slab of five layers within a $3 \times 3$ geometry with the uppermost two layer free to move while the three bottom layers were kept fixed at the bulk geometry. For $\mathrm{Pt}(211)$ and $\mathrm{Pt}(533), 15$-layer and 20-layer slabs, respectively, within a $1 \times 3$ geometry were used with the corresponding numbers of layers free to move. For all considered surfaces, the integration over the first Brillouin zone was replaced by the summation over $3 \times 3 \times 1$ special $k$ points. The (211) surface consists of (111) terraces that are three atom rows wide separated by (100)-like steps. (533) surface are rather similar, the (111) terraces are just four atoms wide.

The AIMD simulations were performed using the Verlet algorithm with a time step of $1 \mathrm{fs}$. The trajectories were started $5 \AA$ above the surface with random lateral position and orientation of the $\mathrm{O}_{2}$ molecules without considering any zero-point vibrational energy in the initial conditions. This is motivated by the fact that the sum of all zero-point energies typically stays approximately constant along the reaction path for adsorption [55]. If not otherwise stated, the simulations were performed within the microcanonical ensemble. However, additional simulations were performed with the second $\mathrm{Pt}$ layer coupled to an Anderson thermostat $[51,56,57]$ with collision rate $0.02 \mathrm{fs}^{-1}$.

\section{RESULTS AND DISCUSSION}

In order to get a first impression of the interaction of $\mathrm{O}_{2}$ with stepped platinum surfaces, we probe the potential energy surface of $\mathrm{O}_{2} / \mathrm{Pt}(211)$ by determining so- 


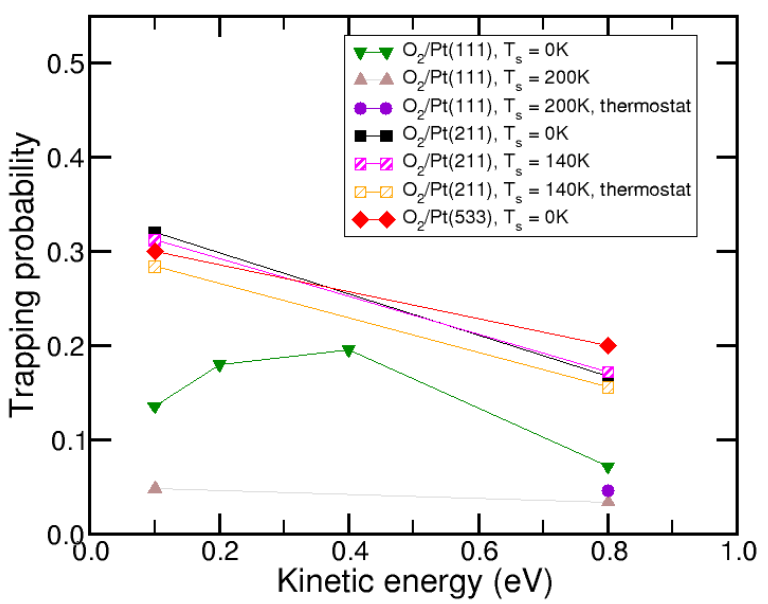

FIG. 2. Trapping probability of $\mathrm{O}_{2}$ impinging on $\mathrm{Pt}(111)$ [51], $\operatorname{Pt}(211)$ and $\operatorname{Pt}(533)$ as a function of the kinetic energy for normal incidence. Computational results derived from AIMD simulations for the surface initially at rest $\left(T_{\mathrm{s}}=0 \mathrm{~K}\right)$, at an initial surface temperature of $T_{\mathrm{s}}=200 \mathrm{~K}$ within the microcanonical ensemble and invoking an Anderson thermostat for the second layer Pt atoms.

called elbow plots [58] and compare them to previous results for the $\mathrm{O}_{2} / \mathrm{Pt}(111)$ system [51] (see Fig. 1). Such elbow plots correspond to two-dimensional cuts through the six-dimensional potential energy surfaces (PESs) as a function of the $\mathrm{O}_{2}$ center of mass distance from the surface and the $\mathrm{O}-\mathrm{O}$ distance with the remaining degrees of freedom kept fixed. In Fig. 1, so-called t-b-t geometries have been considered with the $\mathrm{O}_{2}$ center of mass above a bridge site and the oxygen atoms orientied towards to the adjacent top positions in a flat geometry. On $\mathrm{Pt}(211)$, the $\mathrm{O}_{2}$ center of mass is directly above the atoms at the upper side of the steps with the molecular axis being parallel to the steps.

The pronounced minima $2 \AA$ above the surface with a slightly increased $\mathrm{O}-\mathrm{O}$ bond length of about $1.8 \AA$ correspond to chemisorbed peroxo-like $\left(\mathrm{O}_{2}^{-2}\right)$ molecular states $[59,60]$. Note that the shape of the two PESs is rather similar, however, the depth of the molecular adsorption well differs signigicantly. Above the step sites, molecular adsorption is about $0.7 \mathrm{eV}$ stronger, confirming the results of previous DFT studies [42, 47]. The dissociation channel towards largerO-O distance visible in Fig. 1 does not correspond to the minimum energy path towards dissociation. Still, these calculations also confirm the previous findings that the barriers for $\mathrm{O}_{2}$ dissociation taken as the difference between the energy of the transition state towards dissociative adsorption and the bottom of the molecular adsorption well are almost the same on the flat and the stepped Pt surface [42, 47].

We now turn to the results of the AIMD simulations of $\mathrm{O}_{2}$ molecules impinging on $\mathrm{Pt}(211)$ and $\mathrm{Pt}(533)$. For

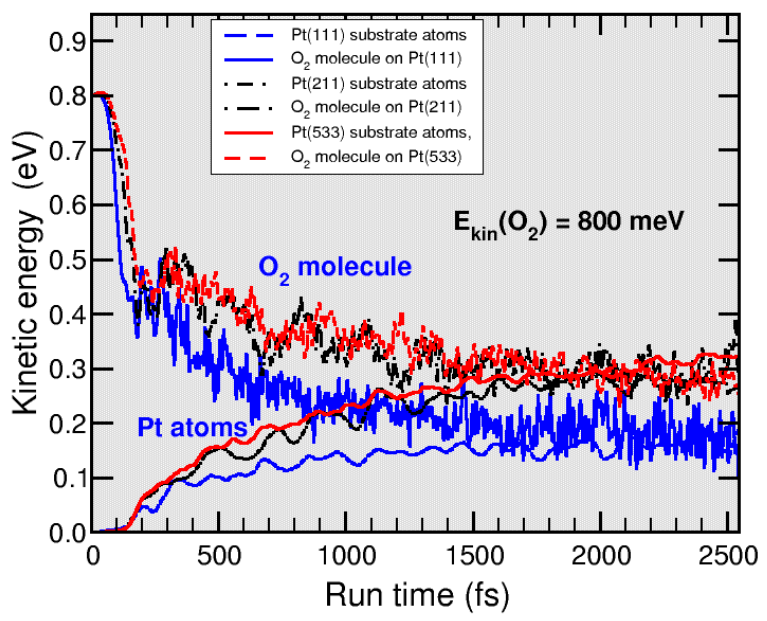

FIG. 3. Kinetic energy redistribution of the $\mathrm{O}_{2}$ molecule and the $\mathrm{Pt}$ substrate atoms of $\mathrm{Pt}(111), \mathrm{Pt}(211)$ and $\mathrm{Pt}(533)$ for an initial $\mathrm{O}_{2}$ kinetic energy of $800 \mathrm{meV}$ averaged over all adsorption events within the microcanonical ensemble for the Pt surface atoms initially at rest corresponding to a surface temperatures of $\mathrm{T}_{s}=0 \mathrm{~K}$.

both surfaces, we have run more than 250 trajectories for each of the two initial kinetic energies $E_{k i n}=0.1 \mathrm{eV}$ and $0.8 \mathrm{eV}$ with the $\mathrm{Pt}$ surface atoms initially at rest which corresponds to an initial surface temperature of $T_{s}=0 \mathrm{~K}$. For $\mathrm{Pt}(211)$, we have additionally run simulations with an initial surface temperature of $T_{s}=0 \mathrm{~K}$ in the microcanonical ensemble, i.e., without invoking any thermostat, and with the Anderson thermostat coupled to the second-layer Pt atoms. The resulting trapping probabulities are plotted in Fig, 2 where we also include the results of AIMD simulations for $\mathrm{O}_{2} \mathrm{Pt}(111)$ [51]. The $\mathrm{O}_{2}$ molecules have considered to be trapped when after at least 2.5 ps run time of the trajectories they have transferred their initial kinetic energy to their internal and the surface degrees of freedom.

First of all we note that the trapping probabilities at the stepped Pt surfaces are larger than on the flat $\mathrm{Pt}(111)$ surface which is in qualitative agreement with experimentally measured trapping probabilities for $\mathrm{O}_{2}$ on $\operatorname{Pt}(533)$ [41] and $\operatorname{Pt}(553)$ [44], confirming the enhanced reactivity of the stepped $\mathrm{Pt}$ surfaces compared to $\mathrm{Pt}(111)$. Quantitatively, the AIMD results for $\mathrm{Pt}(533)$ are lower by almost a factor of two compared to the experiment. This might be a consequence of the fact that the RPBE functional is known to underestimate the interaction of molecules with surfaces, in particular when dispersion effects might play a role $[61,62]$. Note furthermore that apparently surface temperature effects only play a minor role, as far the adsorption of $\mathrm{O}_{2}$ on stepped Pt surfaces is concerned.

In order to understand the energy dissipation of the $\mathrm{O}_{2}$ molecule to the Pt substrate better, in Fig. 3 the mean 

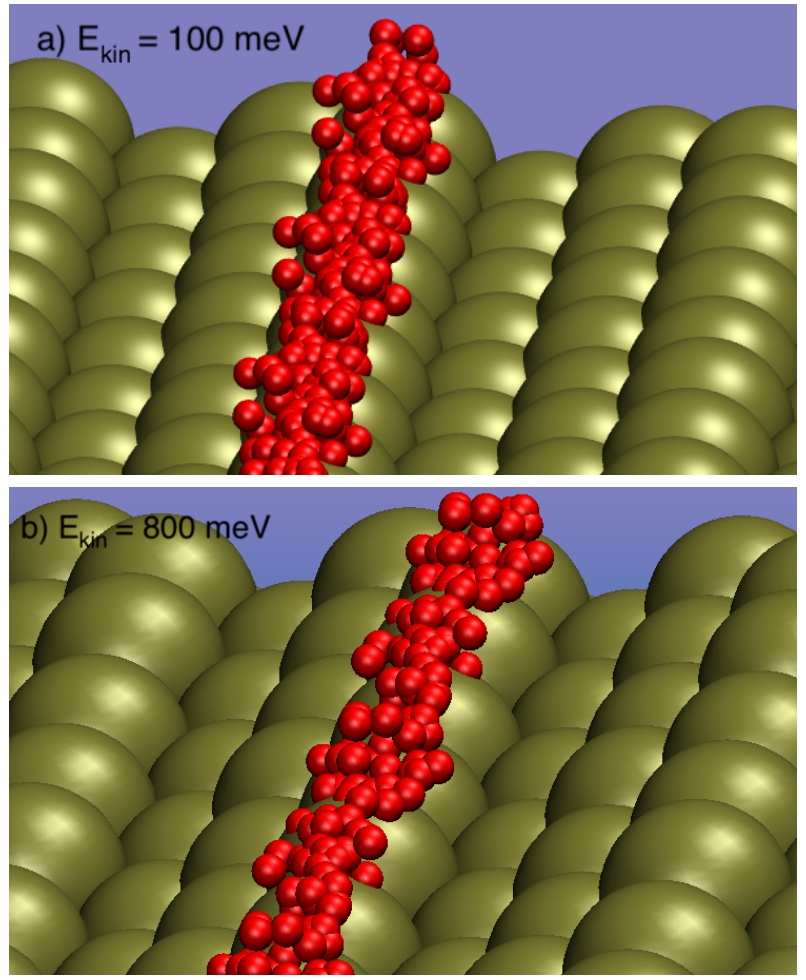

FIG. 4. Spatial distribution of $\mathrm{O}_{2}$ adsorbed molecularly on $\mathrm{Pt}(211)$ after impinging with random impact points within the surface unit cell with initial kinetic energies of the $\mathrm{O}_{2}$ molecules of a) $100 \mathrm{meV}$ and b) $800 \mathrm{meV}$. Note that the periodic images of the oxygen atoms are not plotted for the sake of clarity.

kinetic energies of the impinging $\mathrm{O}_{2}$ molecule with an initial kinetic energy of $0.8 \mathrm{eV}$ and the $\mathrm{Pt}$ substrate atoms of $\mathrm{Pt}(111), \mathrm{Pt}(211)$ and $\mathrm{Pt}(533)$, respectively, are plotted along the run time averaged over all corresponding trapping events. The surface atoms were initially at rest corresponding to a surface temperatures of $\mathrm{T}_{s}=0 \mathrm{~K}$. The larger $\mathrm{O}_{2}$ adsorption energies on $\mathrm{Pt}(211)$ and $\mathrm{Pt}(533)$ compared to $\mathrm{Pt}(111)$ are reflected in the fact that there is a larger energy transfer to the $\mathrm{Pt}$ atoms. Due to the stronger interaction, the $\mathrm{O}_{2}$ molecules become more accelerated when impinging on the stepped surfaces and therefore also transfer more energy to the substrate. The energy distributions for $\mathrm{Pt}(211)$ are rather similar. After about $1.5 \mathrm{ps}$, the mean kinetic energy of the surface atom levels off at a value of about $0.3 \mathrm{eV}$. Considering the equipartition theorem which says that the mean kinetic and the mean potential energy should be the same, one can assume that the $\mathrm{O}_{2}$ molecules have transferred about $0.6 \mathrm{eV}$ to the stepped Pt surface. Still, their mean kinetic energy including vibrational and rotational energy and the motion parallel to the surface is about twice as large as on $\mathrm{Pt}(111)$, indicating that still a substantial amount of internal energy is associated with the trapped molecules.
Next, we consider the spatial distribution of the trapped $\mathrm{O}_{2}$ molecules on $\mathrm{Pt}(211)$ at the end of the trajectories after at least 3 ps which are illustrated in Fig. 4a for an initial $\mathrm{O}_{2}$ kinetic energy of $0.1 \mathrm{eV}$ and in Fig. $4 \mathrm{~b}$ for an initial $\mathrm{O}_{2}$ kinetic energy of $0.1 \mathrm{eV}$. Independent of their initial kinetic energy, all trapped molecules end up at the step sites. This illustrates that the dissipation to the substrate phomons is slow enough that the impinging molecules can still probe the three-atom wide terraces of $\operatorname{Pt}(211)$ and find the energetically most favorable adsorption sites, which is in agreement with exeperimental observations [42]. Note, however, that on Pt(533) with its four-atom wide terraces (not shown in Fig. 4), after 3 ps there are still some trapped molecules located in the middle of the terraces. Apparently, these terraces are already too wide to allow all impinging $\mathrm{O}_{2}$ molecules to directly access the most favorable adsorption sites.

Interestingly enough, about three percent of all $\mathrm{O}_{2}$ molecules impinging on $\mathrm{Pt}(211)$ and $\mathrm{Pt}(533)$ at both considered initial kinetic energies lead to a dissoctive adsorption. In contrast, on $\mathrm{Pt}(111 \mathrm{I}$ no such dissociative $\mathrm{O}_{2}$ adsorption was found in AIMD simulations [51], in agreement with the experiment $[32,33]$. Figure 5a illustrates a dissociative adsorption event of an $\mathrm{O}_{2}$ molecule impinging on $\mathrm{Pt}(211)$ with an initial kinetic energy of $0,8 \mathrm{eV}$. Upon hitting the $\mathrm{Pt}(211)$ surface close to the step edge, the $\mathrm{O}_{2}$ molecule first reorients, the molecule bounces back and forth and starts to vibrate. Eventually, the molecule enters a configuration that is favorable for dissociation and the O-O bond breaks.

A schematic illustration of this process is provided in Figure 5b using representative one-dimensional potential curve. Recall that the barrier for dissociation with respect to the molecular precursor state is of similar height on flat and stepped Pt surfaces [42], but the absolute energetic positions of the molecular precursor state and transition state towards dissociative adsorption are higher for $\mathrm{Pt}(111)$. Note furthermore that in principle a one-dimesional presentation of the potential energy surface is not sufficient to capture the details of the reaction dynamics. In Fig. 5b, there are two schematic trajectories of an $\mathrm{O}_{2}$ molecule impinging on $\mathrm{Pt}(111)$ (black color) and on a stepped Pt surface such as Pt(211) (red color) included. Both particles are assumed to be scattered back from the activation barrier although their nominal energy is higher than the dissociation barrier. However, their particular configurations as far as, e.g., the molecular orientation or the lateral center-of-mass coordinates are concerned, do not correspond to the molecular configuration at the transition state so that these molecules are both initially scattered back upon the first encounter with the surface.

Still, upon impinging on the surface, the approaching $\mathrm{O}_{2}$ molecule does not immediately equilibrate in the precursor well, this typically takes several bounces against the repulsive potential walls. With respect to the gas 

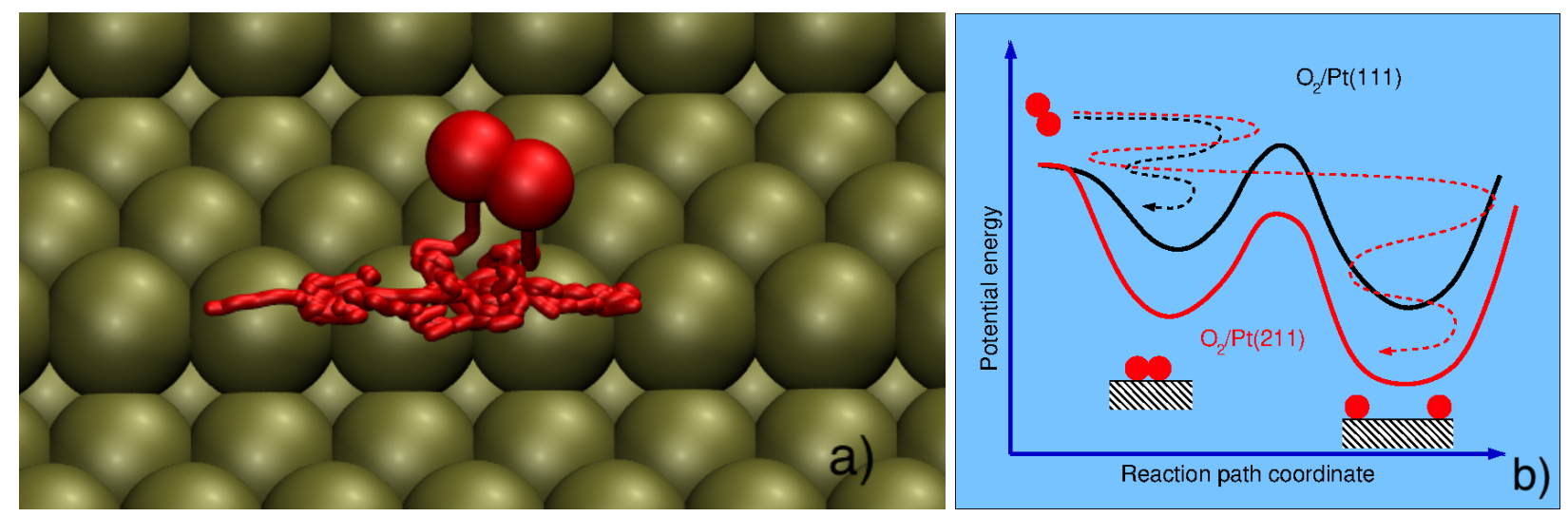

FIG. 5. Dissociative adsorption of $\mathrm{O}_{2}$ on stepped Pt surfaces. a) Traces of an ab initio molecular dynamics trajectory of an $\mathrm{O}_{2}$ molecule impinging on $\mathrm{Pt}(211)$ with an initial kinetic energy of $0,8 \mathrm{eV}$. The periodic images of the oxygen atoms are not plotted for the sake of clarity. b) Schematic drawing of the $\mathrm{O}_{2} / \mathrm{Pt}$ potential energy curves illustrating the hot atom mechanism.

phase level, the absolute barrier for $\mathrm{O}_{2}$ dissociation is higher on the flat $\mathrm{Pt}$ surface compared to the stepped $\mathrm{Pt}$ surfaces. Therefore, at the same kinetic energy the $\mathrm{O}_{2}$ molecule will be much slower upon entering the transition state at $\mathrm{Pt}(111)$, and thus it is more likely that it will be redirected towards the molecular precursor state where is then becomes trapped and eventually equilibrated.

On the stepped surfaces, the topology of the potential energy surface connecting the molecular precursor and the dissociated state might be rather similar. Still, in this region, the potential energy surface is lower in energy with respect to the $\mathrm{O}_{2}$ molecule in the gas phase. Therefore it will be faster in this region which of course also means that the impact upon hitting potential walls might be larger. Yet, even if it rebounces upon the first impact, it might be redirected again towards the surface and keep enough kinetic energy to be able to finally cross the transition state region and become dissociated. So it is the incomplete equilibration in the region of the molecular precursor state which allows the molecules to remain "hot" and keep enough energy to overcome the dissociation barrier.

Note that the time scale of ab initio molecular dynamics simulations is still mich to slow to really model equilibration events. Hence based on this study it cannot be excluded that the thermal equilibration events contribute considerably to the experimentally observed preferential dissociation of $\mathrm{O}_{2}$ at $\mathrm{Pt}$ step sites. Still, the results of this AIMD study provide convincing evidence that a hot precursor mechanism can be operative in the dissociative adsorption of $\mathrm{O}_{2}$ on stepped $\mathrm{Pt}$ surfaces.

\section{CONCLUSIONS}

In this work, I have presented ab initio molecular dynamics simulations of the interaction of $\mathrm{O}_{2}$ molecules with stepped Pt surfaces. The Pt step sites correspond to active sites due to their lower coordination and attract the $\mathrm{O}_{2}$ molecules upon impinging on the surface, as both found in experiments and in the AIMD simulations. Interestingly enough, according to density functional theory calculations, the nominal dissociation barriers on $\mathrm{Pt}$ surfaces determined as the difference between the transition state towards dissociative adsorption and the bottom of the molecular adsorption well are rather similar on flat and stepped Pt surfaces. Still, in the AIMD simulations some direct $\mathrm{O}_{2}$ dissociation events are found at the stepped Pt surfaces, whereas on $\mathrm{Pt}(111)$ no such event is found, even at kinetic energies that are higher than the barrier for dissociative adsorption of $\mathrm{O}_{2}$. The direct dissociative adsorption is caused by the overall more attractive $\mathrm{O}_{2}$ potential energy surface at the stepped surface which can lead to an incomplete equilibration of the $\mathrm{O}_{2}$ molecule upon entering the molecular precursor state so the hot precursor molecule is still able to overcome the activation barrier for dissociative adsorption. The presence of this hot atom chemistry does, however, not preclude that there are also thermally activated $\mathrm{O}_{2}$ dissociation events at the stepped surfaces.

\section{ACKNOWLEDGMENTS}

Financial support has been provided by the German Research Foundation (DFG) through project GR 1503/25-1. These calculations were made possible through a grant of computer time at the John von Neumann Institute for Computing in Jülich. Additional computational resources have been provided by the bwGRiD project and the Justus computer cluster of the bwHPC project of the Federal State of Baden-Württemberg/Germany and the Germany Research Foundation (DFG) through grant number INST 40/575-1. 
[1] B. K. Carpenter, Angew. Chem. Int. Ed. 37, 3340 (1998).

[2] H. Eyring, J. Chem. Phys. 3, 107 (1935).

[3] M. G. Evans and M. Polanyi, Trans. Faraday Soc. 31, 875 (1935).

[4] P. Hänggi, P. Talkner, and M. Borkovec, Rev. Mod. Phys. 62, 251 (1990).

[5] D. G. Truhlar, B. C. Garrett, and S. J. Klippenstein, J. Phys. Chem. 100, 12771 (1996).

[6] H. Kramers, Physica 7, 284 (1940).

[7] H. Brune, J. Wintterlin, R. J. Behm, and G. Ertl, Phys. Rev. Lett. 68, 624 (1992).

[8] J. Wintterlin, R. Schuster, and G. Ertl, Phys. Rev. Lett. 77, 123 (1996).

[9] L. C. Ciacchi and M. C. Payne, Phys. Rev. Lett. 92, 176104 (2004).

[10] M. L. Neuburger and D. P. Pullman, J. Chem. Phys. 113, 1249 (2000).

[11] A. J. Komrowski, J. Z. Sexton, K. A. C., M. Binetti, O. Weisse, and E. Hasselbrink, Phys. Rev. Lett. 87, 246103 (2001).

[12] C. Carbogno, J. Behler, A. Groß, and K. Reuter, Phys. Rev. Lett. 101, 096104 (2008).

[13] C. Carbogno, J. Behler, K. Reuter, and A. Groß, Phys. Rev. B 81, 035410 (2010).

[14] L. Österlund, I. Zorić, and B. Kasemo, Phys. Rev. B 55, 15452 (1997).

[15] G.-J. Kroes, Phys. Chem. Chem. Phys. 23, 8962 (2021).

[16] C. Sendner and A. Groß, J. Chem. Phys. 127, 014704 (2007).

[17] A. Groß, Phys. Rev. Lett. 103, 246101 (2009).

[18] J. Meyer and K. Reuter, Angew. Chem. Int. Ed. 53, 4721 (2014).

[19] V. J. Bukas, S. Mitra, J. Meyer, and K. Reuter, J. Chem. Phys. 143 (2015), 10.1063/1.4926989.

[20] V. J. Bukas and K. Reuter, Phys. Rev. Lett. 117, 146101 (2016).

[21] B. Jiang and H. Guo, J. Chem. Phys. 150, 180901 (2019).

[22] S. P. Rittmeyer, D. J. Ward, P. Gütlein, J. Ellis, W. Allison, and K. Reuter, Phys. Rev. Lett. 117, 196001 (2016).

[23] S. P. Rittmeyer, V. J. Bukas, and K. Reuter, Adv. Phys. X 3, 1381574 (2018).

[24] C. L. Box, Y. Zhang, R. Yin, B. Jiang, and R. J. Maurer, JACS Au 1, 164 (2021).

[25] H.-J. Freund, Surf. Sci. 500, 271 (2002).

[26] H. A. Gasteiger, S. S. Kocha, B. Sompalli, and F. T. Wagner, Appl. Catal. B 56, 9 (2005).

[27] Y. Gohda, S. Schnur, and A. Groß, Faraday Discuss. 140, 233 (2008).

[28] A. Groß, F. Gossenberger, X. Lin, M. Naderian, S. Sakong, and T. Roman, J. Electrochem. Soc. 161, E3015 (2014).

[29] S. Sakong, D. Mahlberg, T. Roman, M. Pandey, and A. Groß, J. Phys. Chem. C 124, 27604 (2020).

[30] X. Zhang, H. Li, Z. Xia, S. Yu, S. Wang, and G. Sun, Phys. Chem. Chem. Phys. 23, 1584 (2021).

[31] W. Wurth, J. Stöhr, P. Feulner, X. Pan, K. R. Bauchspiess, Y. Baba, E. Hudel, G. Rocker, and D. Menzel, Phys. Rev. Lett. 65, 2426 (1990).

[32] C. T. Rettner and C. B. Mullins, J. Chem. Phys. 94, 1626 (1991).
[33] P. D. Nolan, B. R. Lutz, P. L. Tanaka, J. E. Davis, and C. B. Mullins, Phys. Rev. Lett. 81, 3179 (1998).

[34] A. Groß, A. Eichler, J. Hafner, M. J. Mehl, and D. A. Papaconstantopoulos, Surf. Sci. 539, L542 (2003).

[35] A. Groß, A. Eichler, J. Hafner, M. J. Mehl, and D. A. Papaconstantopoulos, J. Chem. Phys. 124, 174713 (2006).

[36] S. Dahl, A. Logadottir, R. C. Egeberg, J. H. Larsen, I. Chorkendorff, E. Törnqvist, and J. K. Nørskov, Phys. Rev. Lett. 83, 1814 (1999).

[37] B. Hammer, Phys. Rev. Lett 83, 3681 (1999).

[38] A. Groß, J. Comput. Theor. Nanosci. 5, 894 (2008).

[39] C. Zhang, B. Wang, A. Hellman, M. Shipilin, A. Schaefer, L. R. Merte, S. Blomberg, X. Wang, P.-A. Carlsson, E. Lundgren, J. Weissenrieder, A. Resta, A. Mikkelsen, J. N. Andersen, and J. Gustafson, Surf. Sci. 715, 121928 (2022).

[40] P. J. Feibelman, S. Esch, and T. Michely, Phys. Rev. Lett. 77, 2257 (1996).

[41] A. T. Gee and B. E. Hayden, J. Chem. Phys. 113, 10333 (2000).

[42] P. Gambardella, Z̆. S̆ljivančanin, B. Hammer, M. Blanc, K. Kuhnke, and K. Kern, Phys. Rev. Lett. 87, 056103 (2001).

[43] M. J. Kolb, F. Calle-Vallejo, L. B. F. Juurlink, and M. T. M. Koper, JCP 140, 134708 (2014).

[44] L. Jacobse, A. den Dunnen, and L. B. F. Juurlink, J. Chem. Phys. 143, 014703 (2015).

[45] C. Badan, R. G. Farber, Y. Heyrich, M. T. M. Koper, D. R. Killelea, and L. B. F. Juurlink, J. Phys. Chem. C 120, 22927 (2016).

[46] K. Cao, R. van Lent, A. W. Kleyn, M. Kurahashi, and L. B. F. Juurlink, Proc. Natl. Acad. Sci. 116, 13862 (2019).

[47] Z̆. S̆ljivančanin and B. Hammer, Surf. Sci. 515, 235 (2002).

[48] T. Ogawa, A. Kuwabara, C. A. J. Fisher, H. Moriwake, and T. Miwa, J. Phys. Chem. C 117, 9772 (2013).

[49] G. Kresse and J. Furthmüller, Phys. Rev. B 54, 11169 (1996).

[50] B. Hammer, L. B. Hansen, and J. K. Nørskov, Phys. Rev. B 59, 7413 (1999).

[51] A. Groß, Catal. Today 260, 60 (2016).

[52] J. P. Perdew, K. Burke, and M. Ernzerhof, Phys. Rev. Lett. 77, 3865 (1996).

[53] P. E. Blöchl, Phys. Rev. B 50, 17953 (1994).

[54] G. Kresse and D. Joubert, Phys. Rev. B 59, 1758 (1999).

[55] A. Groß and M. Scheffler, J. Vac. Sci. Technol. A 15, 1624 (1997).

[56] H. C. Andersen, J. Chem. Phys. 72, 2384 (1980).

[57] A. L. L. East, T. Bucko, and J. Hafner, J. Chem. Phys. 131, 104314 (2009).

[58] G.-J. Kroes, A. Groß, E. J. Baerends, M. Scheffler, and D. A. McCormack, Acc. Chem. Res. 35, 193 (2002).

[59] A. Eichler and J. Hafner, Phys. Rev. Lett. 79, 4481 (1997).

[60] A. Eichler, F. Mittendorfer, and J. Hafner, Phys. Rev. B 62, 4744 (2000).

[61] J. Wellendorff, T. L. Silbaugh, D. Garcia-Pintos, J. K. Nørskov, T. Bligaard, F. Studt, and C. T. Campbell, Surf. Sci. 640, 36 (2015).

[62] D. Mahlberg, S. Sakong, K. Forster-Tonigold, and A. Groß, J. Chem. Theory Comput. 15, 3250 (2019). 